\title{
Ventilator-derived dynamic respiratory system compliance : Comparison with static compliance in children
}

\section{Kaskinen, Anu K.}

2018-02

Kaskinen , A K , Kirjavainen , T, Rautiainen , P , Martelius , L , Andersson , S \& Pitkänen , O M 2018 , ' Ventilator-derived dynamic respiratory system compliance : Comparison with static compliance in children ' , Respiratory Physiology \& Neurobiology , vol. 249 , pp. 32-34 . https://doi.org/10.1016/j.resp.2017.12.007

http://hdl.handle.net/10138/300881

https://doi.org/10.1016/j.resp.2017.12.007

publishedVersion

Downloaded from Helda, University of Helsinki institutional repository.

This is an electronic reprint of the original article.

This reprint may differ from the original in pagination and typographic detail.

Please cite the original version. 
Short communication

\title{
Ventilator-derived dynamic respiratory system compliance: Comparison with static compliance in children
}

\author{
Anu K. Kaskinen ${ }^{\mathrm{a}, \mathrm{b}, *}$, Turkka Kirjavainen ${ }^{\mathrm{a}}$, Paula Rautiainen ${ }^{\mathrm{a}}$, Laura Martelius ${ }^{\mathrm{c}}$, Sture Andersson ${ }^{\mathrm{a}}$, \\ Olli M. Pitkänen ${ }^{\mathrm{a}}$ \\ a University of Helsinki and Helsinki University Hospital, Children's Hospital, Divisions of Pediatric Cardiology, Anesthesia and Intensive Care, and Neonatology, Finland \\ ${ }^{\mathrm{b}}$ Päijät-Häme Central Hospital, Department of Pediatrics, Finland

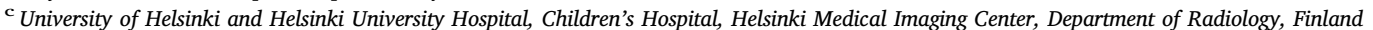

\section{A R T I C L E I N F O}

\section{Keywords:}

Compliance

Congenital heart disease

Lung physiology

Mechanical ventilation

Pulmonary edema

\begin{abstract}
A B S T R A C T
Measurement of dynamic lung compliance during breathing requires measurement of esophageal pressure, whereas static respiratory system compliance (Crs) method requires several airway occlusions. Despite their precision these compliance methods are cumbersome and not suitable for evaluation of pulmonary system in intensive care. The current ventilators display dynamic Crs, which, however, is seldom utilized in clinical practice. We studied the feasibility of ventilator-derived dynamic Crs measurement in pulmonary evaluation after congenital cardiac surgery in children. In 50 children static Crs was measured by double-occlusion technique, and compared with simultaneous ventilator-derived dynamic Crs values. The early postoperative dynamic and static Crs showed a correlation $(r=0.57, \mathrm{p}<0.0001)$, but static Crs was $48 \%$ higher than dynamic ( $\mathrm{p}<0.0001)$. Dynamic Crs measurement showed no correlation with radiographic lung edema findings, whereas the static Crs showed a negative correlation with radiographic lung edema scoring $(r=-0.50$, $\mathrm{p}=0.0002$ ). Thus ventilator-derived dynamic Crs seems less reliable in postoperative pulmonary evaluation than static Crs.
\end{abstract}

\section{Introduction}

Lung compliance or respiratory system compliance (Crs) can be measured in static airways with halted airflow, or as dynamic compliance during breathing. Currently, the best estimate of compliance is acquired with airway occlusions for static Crs, or with esophageal pressure measurement as an estimate of pleural pressure for dynamic lung compliance measurement (Gerhardt et al., 1989; Goetz et al., 2001). However, due to their laborious and cumbersome nature, neither compliance measurement method lends itself to daily clinical practice in pediatric intensive care.

Today's modern ventilators, without measuring esophageal pressure, continuously display dynamic expiratory Crs, which has shown a strong correlation with static Crs in neonates with respiratory failure (Kugelman et al., 1995; Storme et al., 1992). Moreover, dynamic lung mechanics may be useful in optimizing ventilator management in critically ill patients (Macnaughton 2006; Stenqvist et al., 2008).

We studied the feasibility of ventilator-derived dynamic Crs measurement by comparing it with static Crs during expiration in children without respiratory failure after congenital cardiac surgery. Additionally, we studied whether early postoperative dynamic and static Crs reflect chest radiography (CXR) lung edema assessment, or short-term clinical outcome.

\section{Methods}

We studied 50 children $1-6 \mathrm{~h}$ after congenital cardiac surgery at Children's Hospital, Helsinki University Hospital (Table 1). The institution's Ethics Committee approved the study protocol, and parents provided written informed consent. The complexity of care and potential for postoperative morbidity was defined according to the Aristotle Comprehensive Complexity (ACC) score, and short-term clinical outcome was interpreted as length of mechanical ventilation and pediatric intensive care unit (PICU) stay (Table 1).

All patients had a cuffed endotracheal tube and were mechanically normoventilated with pressure-controlled synchronized intermittent mandatory ventilation (SIMV) by the SERVO-i ventilator (Maquet, Rastatt, Germany). The expiratory static Crs was measured by the double-occlusion technique (Labmanager 4-521; Erich Jaeger GmbH, Hoechberg, Germany) 1-6h postoperatively in PICU (Goetz et al., 2001). Airflow was occluded on average for $413 \pm 201 \mathrm{~ms}$ during the first occlusion, for $253 \pm 94 \mathrm{~ms}$

\footnotetext{
* Corresponding author at: Pediatric Research Center, University of Helsinki and Helsinki University Hospital, Biomedicum Helsinki 2U, 00290 Helsinki, Finland.

E-mail address: anu.kaskinen@helsinki.fi (A.K. Kaskinen).
} 
Table 1

Clinical characteristics, ventilator and static Crs measurement settings.

\begin{tabular}{|c|c|}
\hline Age [months] & $4.7(1.4-10.7)$ \\
\hline \multicolumn{2}{|l|}{ Diagnoses with operations } \\
\hline AVSD \& TOF, repair & $1(2 \%)$ \\
\hline CoA, repair of LVOTO & $2(4 \%)$ \\
\hline Critical Pulmonary Stenosis, repair & $1(2 \%)$ \\
\hline Double Aortic Arch, repair in thoracotomy & $1(2 \%)$ \\
\hline $\begin{array}{l}\text { Intracardiac L-R Shunt, repair by sutures or patching in ASD } \\
\text { (2), ASD\&VSD (4), AVSD (4), VSD (8) }\end{array}$ & $18(36 \%)$ \\
\hline Patent Ductus Arteriosus, repair & $1(2 \%)$ \\
\hline $\begin{array}{l}\text { PA + VSD/TOF, repair (4), closure of residual VSD (1), LPA \& } \\
\text { RPA plasty (1) }\end{array}$ & $6(12 \%)$ \\
\hline $\begin{array}{l}\text { Single Ventricle; Norwood procedure (1), BDG (4), TCPC (4), } \\
\text { PA banding (1) }\end{array}$ & $10(20 \%)$ \\
\hline TAPVD, repair & $1(2 \%)$ \\
\hline TGA, arterial switch operation & $6(12 \%)$ \\
\hline Truncus Arteriosus, repair & $2(4 \%)$ \\
\hline $\begin{array}{l}\text { RVOTO \& SVAS, repair of RVOTO by resection and SVAS by } \\
\text { patch augmentation }\end{array}$ & $1(2 \%)$ \\
\hline Aristotle comprehensive complexity score & $8.8(6.0-11.0)$ \\
\hline Delayed sternal closure & $7(14 \%)$ \\
\hline \multicolumn{2}{|l|}{ Cardiopulmonary bypass } \\
\hline Perfusion time in minutes & $82(54-148)$ \\
\hline Aortic cross-clamping in minutes & $46(16-93)$ \\
\hline Days in PICU postoperatively & $3(2-6)$ \\
\hline Days on mechanical ventilation & $1(0.5-3)$ \\
\hline \multicolumn{2}{|l|}{ Ventilator settings } \\
\hline PEEP & $5(5-5)$ \\
\hline Pressure support over PEEP & $14(13-16)$ \\
\hline SIMV frequency per minute & $25(20-30)$ \\
\hline Tidal volume [ml] & $60(36-102)$ \\
\hline \multicolumn{2}{|l|}{ Static Crs measurement } \\
\hline First occlusion [ms] & $413 \pm 201$ \\
\hline Second occlusion [ms] & $253 \pm 94$ \\
\hline First pressure plateau [ms] & $250 \pm 120$ \\
\hline Second pressure plateau [ms] & $150 \pm 70$ \\
\hline
\end{tabular}

$\mathrm{ASD}=$ atrial septal defect, $\mathrm{AVSD}=$ atrioventricular septal defect, $\mathrm{BDG}=$ bidirectional Glenn procedure, $\mathrm{CoA}=$ coarctation of Aorta, LPA $=$ left pulmonary artery, $\mathrm{IQR}=$ interquartile range, $\mathrm{L}-\mathrm{R}=$ left to right, $\mathrm{PA}=$ pulmonary atresia, $\mathrm{PEEP}=$ positive end expiratory pressure, PICU = pediatric intensive care unit, RPA = right pulmonary artery, RVOTO = right ventricular outflow tract obstruction, SIMV = synchronized intermittent mandatory ventilation, SVAS $=$ supravalvular aortic stenosis, TAPVD $=$ total anomalous pulmonary venous drainage, TCPC $=$ total cavopulmonary connection, $\mathrm{TGA}=$ transposition of the great arteries, TOF $=$ tetralogy of Fallot, VSD $=$ ventricular septal defect

Data are presented as $\mathrm{n}(\%)$, median with interquartile range or mean $\pm \mathrm{SD}$, as appropriate.

during the second occlusion, and a minimum of 10 respiratory cycles occurred between each double occlusion (Table 1). On average five static Crs values [coefficient of variation 0.06] were available for each patient. One patient's pressure-volume curves lacked acceptable pressure plateaus, thus these data were omitted from analysis. The ventilator and static Crs measurement settings are provided in Table 1. The SERVO-i calculated the expiratory dynamic Crs for each breath as follows:

dyn $C r s=\frac{\text { expiratory tidal volume }}{\text { end inspiratory pressure }- \text { end expiratory pressure }}$

This formula is comparable to multiple ventilators on market. Without airflow occlusions Servo-i measures end inspiratory pressure just before start of expiration when inspiratory airflow diminishes to almost zero, and end expiratory pressure just before start of inspiration. Thus, end expiratory pressure is close to positive end expiratory pressure (PEEP). The average value for a 15-min period coinciding with the static Crs measurement was used for analyses. The CXRs taken within $2 \mathrm{~h}$ of static Crs measurement were scored for lung edema on a 4-step scale $(0=$ normal lung, $1=$ minimal opacity not obscuring lung vessels, 2 = opacity partially obscuring lung vessels, $3=$ opacity totally obscuring lung vessels).
Our primary interest was to study the correlation between dynamic and static Crs. The sample size calculation based on correlations with $r$ being up to 0.90 found in previous studies on neonates would have allowed sample size around 10 patients (Kugelman et al., 1995; Storme et al., 1992). However, we wanted to base sample size calculation on a lower correlation $(r=0.65)$ because we estimated lower $r$ to be more realistic but still clinically significant. To find a correlation between 0.5 and 0.7 , we needed 42 patients (based on $80 \%$ power and a two-sided test with $\alpha=0.05$ ) (3). Additional patients were recruited to allow for dropouts.

Variables on a qualitative scale are presented as n with percentages. Normality of variables on a continuous scale was assessed visually and by Kolmogorov-Smirnov test. Variables on a continuous scale described as mean $\pm \mathrm{SD}$ or median with interquartile range (IQR) were compared with Student's $t$ test or Mann-Whitney $U$ test, as appropriate. Correlations were examined with Pearson's test and linear regression analysis or Spearman's test, as appropriate. We further analyzed difference between dynamic and static Crs by Bland-Altman method. In the Bland-Altman plot the difference of the dynamic and static Crs was plotted on $\mathrm{y}$-axis against the mean of the two Crs measurements on $\mathrm{x}$ axis. A $p$ value $\leq 0.05$ was considered significant for all statistical analyses. Statistical analyses were performed with SPSS 21.0 (IBM Corp., Armonk, NY, USA) and Prism 7.0 (GraphPad Software, La Jolla, CA, USA).

\section{Results}

Dynamic and static Crs demonstrated a positive correlation $(r=0.57$, $\mathrm{p}<0.0001$ ) (Fig. 1A), but static Crs was $48 \%$ higher than dynamic $(7.4 \pm 2.4 \mathrm{ml} / \mathrm{kPa} / \mathrm{kg}$ vs. $5.0 \pm 1.4 \mathrm{ml} / \mathrm{kPa} / \mathrm{kg}, \mathrm{p}<0.0001)$. In BlandAltman analysis, the mean difference between static and dynamic Crs was $2.50 \mathrm{ml} / \mathrm{kPa} / \mathrm{kg}$ (Fig. 1B).

Dynamic Crs measurement showed no correlation with CXR LE scoring, whereas static Crs showed a negative correlation $(r=-0.50$, $\mathrm{p}=0.0002)$ (Fig. 2). Both dynamic Crs $(\mathrm{p}=0.42)$ and static Crs $(\mathrm{p}=0.17$ ) showed no difference between patients with open or closed sternum. No significant correlations occurred between dynamic or static Crs and length of cardiopulmonary bypass (CPB), length of aortic cross-clamping, ACC score, or short-term clinical outcome interpreted as length of mechanical ventilation and PICU stay (data not shown). Both dynamic $(r=0.41, \mathrm{p}=0.006)$ and static Crs. $(\mathrm{r}=0.44$, $\mathrm{p}=0.002$ ) correlated positively with tidal volume, but not with PEEP, pressure support over PEEP, or respiratory rate (data not shown).

\section{Discussion}

We found a moderate correlation between dynamic and static Crs. However, the correlation was weaker than demonstrated by previous studies on newborn infants (Kugelman et al., 1995; Storme et al., 1992). The distinction in these correlations may derive from differences in static Crs methods, and in study patients. The previous studies used single occlusion technique, which is influenced by airway and tracheal tube resistance, similarly to dynamic Crs (Kugelman et al., 1995; Storme et al., 1992). In the present study, we chose the double-occlusion technique, which calculates Crs as the difference between two occlusions' pressure-volume pairs, and is unaffected by airway resistance (Goetz et al., 2001). Furthermore, our patients without a pulmonary source for respiratory failure were homogenous in terms of their ventilatory state.

Bland-Altman analysis clearly demonstrated that dynamic and static Crs values are not equivalent. Despite correlating, dynamic Crs values were significantly lower than corresponding static Crs, which is consistent with previous studies (Kugelman et al., 1995; Stenqvist et al., 2008). These measurements may assess different phenomena, since dynamic Crs is measured during some airflow and without airflow occlusions. Thus, dynamic Crs is influenced by the effect of simultaneous 
A

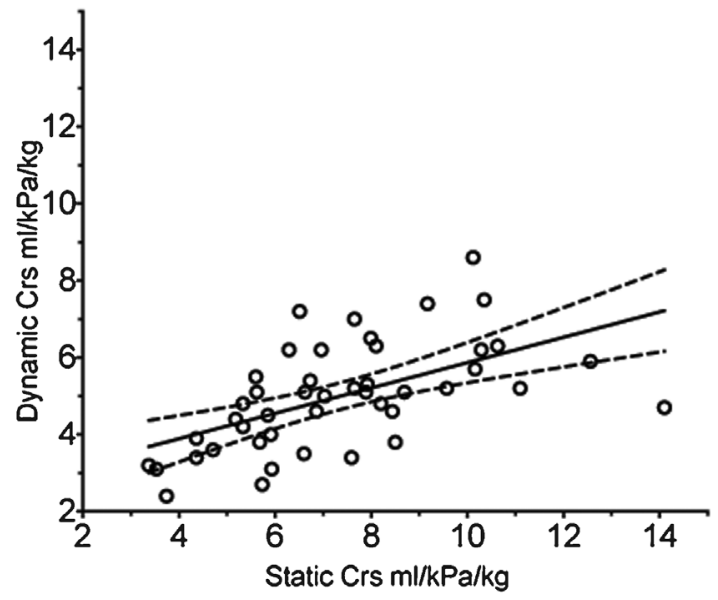

B

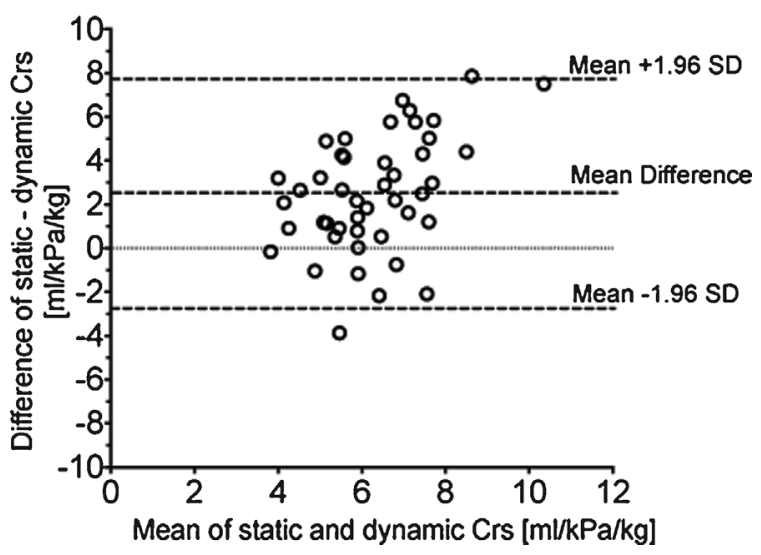

Fig. 1. During regular breathing static respiratory system compliance (Crs) correlated with simultaneously determined dynamic Crs $(r=0.57, \mathrm{p}<0.0001)(\mathrm{A})$. Dashed lines indicate the $95 \%$ confidence interval. In Bland-Altman analysis (B), the mean difference between static and dynamic Crs was $2.50 \mathrm{ml} / \mathrm{kPa} / \mathrm{kg}$ and $95 \%$ of the differences fell between -2.71 and $7.71 \mathrm{ml} / \mathrm{kPa} / \mathrm{kg}$.

resistance. Furthermore, only static Crs correlated with CXR lung edema scoring. Consequently, static Crs may reflect the postoperative state of lung parenchyma and possible effect of increased extravascular lung water better than ventilator-derived dynamic Crs.

Although CPB and cardiac surgery may reduce postoperative Crs values (Lanteri et al., 1995; Polese et al., 1999), we found no correlation between Crs and length of CPB or aortic cross clamping. Neither did we find correlation between early postoperative Crs and short-term outcome. Although Crs has associated with short-term outcome in adults with acute lung injury (Seeley et al., 2011), in children undergoing congenital cardiac surgery the length of mechanical ventilation and PICU stay may be more related to multiple factors during the postoperative course (Brown et al., 2003).

In conclusion, despite reasonable correlation between the dynamic and static Crs, the values do differ, and only static Crs correlates with radiographic findings of lung edema. Therefore ventilator-derived dynamic Crs seems less reliable in assessment of postoperative lung parenchyma than static Crs.

\section{Conflict of interest and source of funding}

Supported by the Aarne Koskelo Foundation, the Academy of Finland, the Finnish Medical Foundation, the Foundation for Pediatric
A

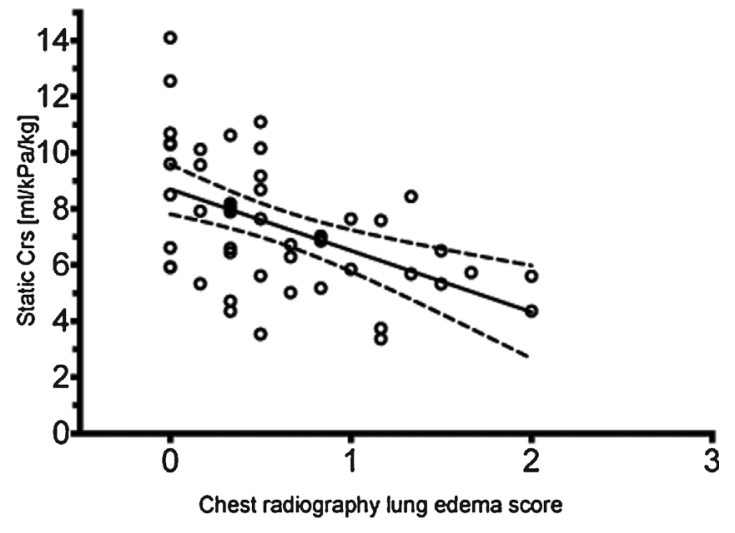

B

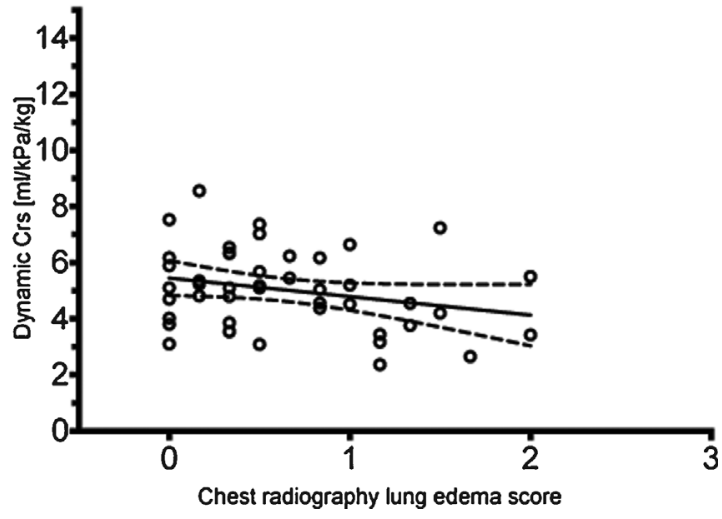

Fig. 2. Early postoperative chest radiography lung edema scoring showed a significant linear correlation with static respiratory system compliance $(r=-0.50, p=0.0002)(A)$, but not with dynamic respiratory system compliance $(r=0.26, p=0.08)(B)$. Dashed lines indicate the $95 \%$ confidence interval.

Research, and the Tor och Kirsti Johansson Foundation. The authors declare that they have no conflict of interest.

\section{References}

Brown, K.L., Ridout, D.A., Goldman, A.P., Hoskote, A., Penny, D.J., 2003. Risk factors for long intensive care unit stay after cardiopulmonary bypass in children. Crit. Care Med. 31 (1), 28-33.

Gerhardt, T., Reifenberg, L., Duara, S., Bancalari, E., 1989. Comparison of dynamic and static measurements of respiratory mechanics in infants. J. Pediatr. 114 (1), 120-125.

Goetz, I., Hoo, A.F., Lum, S., Stocks, J., 2001. Assessment of passive respiratory mechanics in infants: double versus single occlusion? Eur. Respir. J. 17 (3), 449-455.

Kugelman, A., Keens, T.G., deLemos, R., Durand, M., 1995. Comparison of dynamic and passive measurements of respiratory mechanics in ventilated newborn infants. Pediatr. Pulmonol. 20 (4), 258-264.

Lanteri, C.J., Kano, S., Duncan, A.W., Sly, P.D., 1995. Changes in respiratory mechanics in children undergoing cardiopulmonary bypass. Am. J. Respir. Crit. Care Med. 6 (Pt. 1), 1893-1900.

Macnaughton, P.D., 2006. New ventilators for the ICU-usefulness of lung performance reporting. Br. J. Anaesth. 97 (1), 57-63.

Polese, G., Lubli, P., Mazzucco, A., Luzzani, A., Rossi, A., 1999. Effects of open heart surgery on respiratory mechanics. Intensive Care Med. 25 (10), 1092-1099.

Seeley, E.J., McAuley, D.F., Eisner, M., Miletin, M., Zhuo, H., Matthay, M.A., Kallet, R.H., 2011. Decreased respiratory system compliance on the sixth day of mechanical ventilation is a predictor of death in patients with established acute lung injury. Respir. Res. 12, 52.

Stenqvist, O., Odenstedt, H., Lundin, S., 2008. Dynamic respiratory mechanics in acute lung injury/acute respiratory distress syndrome: research or clinical tool? Curr. Opin. Crit. Care 14 (1), 87-93.

Storme, L., Riou, Y., Leclerc, F., Kacet, N., Dubos, J.P., Gremillet, C., Rousseau, S., Lequien, P., 1992. Respiratory mechanics in mechanically ventilated newborns: a comparison between passive inflation and occlusion methods. Pediatr. Pulmonol. 12 (4), 203-212. 